This item was submitted to Loughborough's Research Repository by the author.

Items in Figshare are protected by copyright, with all rights reserved, unless otherwise indicated.

\title{
Viscous placebo and carbohydrate breakfasts similarly decrease appetite and increase resistance exercise performance compared with a control breakfast in trained males
}

\section{PLEASE CITE THE PUBLISHED VERSION}

https://doi.org/10.1017/S0007114520001002

\section{PUBLISHER}

Cambridge University Press

\section{VERSION}

VoR (Version of Record)

\section{PUBLISHER STATEMENT}

This article has been published in British Journal of Nutrition http://doi.org/10.1017/S0007114520001002. This version is published under a Creative Commons CC-BY-NC-ND. No commercial re-distribution or re-use allowed. Derivative works cannot be distributed. (C) The Authors.

\section{LICENCE}

CC BY-NC-ND 4.0

\section{REPOSITORY RECORD}

Naharudin, MN, J Adams, H Richardson, T Thomson, C Oxinou, C Marshall, DJ Clayton, et al.. 2020. "Viscous Placebo and Carbohydrate Breakfasts Similarly Decrease Appetite and Increase Resistance Exercise Performance Compared with a Control Breakfast in Trained Males". Loughborough University. https://hdl.handle.net/2134/13234901.v1. 


\title{
Viscous placebo and carbohydrate breakfasts similarly decrease appetite and increase resistance exercise performance compared with a control breakfast in trained males
}

\author{
M. N. Naharudin ${ }^{1,2}$, J. Adams ${ }^{1}$, H. Richardson ${ }^{1}$, T. Thomson ${ }^{1}$, C. Oxinou ${ }^{1}$, C. Marshall ${ }^{1}$, D. J. Clayton ${ }^{1,3}$, \\ S. A. Mears ${ }^{1}$, A. Yusof ${ }^{2}$, C. J. Hulston ${ }^{1}$ and L. J. James ${ }^{1 *}$ \\ ${ }^{1}$ National Centre for Sport and Exercise Medicine, School of Sport Exercise and Health Science, Loughborough University, \\ Loughborough LE11 3TU, UK \\ ${ }^{2}$ Centre for Sports and Exercise Sciences, University of Malaya, 50603 Kuala Lumpur, Malaysia \\ ${ }^{3}$ School of Science and Technology, Nottingham Trent University, Nottingham NG11 8NS, UK
}

(Submitted 26 November 2019 - Final revision received 3 February 2020 - Accepted 19 February 2020 - First published online 16 March 2020)

Abstract

Given the common view that pre-exercise nutrition/breakfast is important for performance, the present study investigated whether breakfast influences resistance exercise performance via a physiological or psychological effect. Twenty-two resistance-trained, breakfast-consuming men completed three experimental trials, consuming water-only (WAT), or semi-solid breakfasts containing $0 \mathrm{~g} / \mathrm{kg}$ (PLA) or $1.5 \mathrm{~g} / \mathrm{kg}$ (CHO) maltodextrin. PLA and CHO meals contained xanthan gum and low-energy flavouring (approximately $122 \mathrm{~kJ}$ ), and subjects were told both 'contained energy'. At $2 \mathrm{~h}$ post-meal, subjects completed four sets of back squat and bench press to failure at $90 \%$ ten repetition maximum. Blood samples were taken pre-meal, $45 \mathrm{~min}$ and $105 \mathrm{~min}$ post-meal to measure serum/plasma glucose, insulin, ghrelin, glucagon-like peptide-1 and peptide tyrosine-tyrosine concentrations. Subjective hunger/fullness was also measured. Total back squat repetitions were greater in $\mathrm{CHO}$ (44 (sD 10) repetitions) and PLA ( 43 (SD 10) repetitions) than WAT (38 (SD 10) repetitions; $P<0.001$ ). Total bench press repetitions were similar between trials (WAT 37 (SD 7) repetitions; $\mathrm{CHO} 39$ (SD 7) repetitions; PLA 38 (SD 7) repetitions; $P=0 \cdot 130$ ). Performance was similar between CHO and PLA trials. Hunger was suppressed and fullness increased similarly in PLA and CHO, relative to WAT $(P<0 \cdot 001)$. During CHO, plasma glucose was elevated at $45 \mathrm{~min}(P<0.05)$, whilst serum insulin was elevated $(P<0.05)$ and plasma ghrelin suppressed at 45 and $105 \mathrm{~min}$ $(P<0.05)$. These results suggest that breakfast/pre-exercise nutrition enhances resistance exercise performance via a psychological effect, although a potential mediating role of hunger cannot be discounted.

Key words: Weight training: Strength: Nocebo effect: Fasting: Ghrelin

Resistance exercise is regularly performed by athletes/recreational exercisers, and performance in such sessions might have important implications for training volume and consequently gains in muscle mass/strength ${ }^{(1)}$, as well as for prevention of/recovery from injury. The pre-exercise meal, particularly its carbohydrate content, is an important component of an athlete's nutrition plan ${ }^{(2)}$, with current guidelines recommending $1-4 \mathrm{~g}$ carbohydrate/kg body mass should be consumed in the $1-4 \mathrm{~h}$ pre-exercise ${ }^{(3)}$. Previous research has demonstrated that consumption of carbohydrate in the hours before endurance exercise enhances performance ${ }^{(2)}$, but little is known about how such nutrition strategies influence performance in resistancetype exercise.
Carbohydrate intake at the first meal of the day following an overnight fast (i.e. breakfast) increases both liver ${ }^{(4)}$ and muscle ${ }^{(5,6)}$ glycogen. Muscle glycogen appears to be an important fuel source for resistance-type exercise, with a single exercise bout reducing muscle glycogen content by up to $40 \%{ }^{(7,8)}$. Some have suggested that this muscle glycogen depletion might play a role in the development of fatigue during resistance exercise ${ }^{(9,10)}$. Consequently, the elevation of endogenous glycogen stores through pre-exercise feeding might delay fatigue and enhance performance ${ }^{(11)}$. Indeed, commencing a bout of resistance exercise with reduced glycogen stores has been shown to reduce performance by some ${ }^{(12-14)}$, but not all ${ }^{(15)}$ studies. Similarly, we recently

Abbreviations: $\mathrm{CHO}$, carbohydrate; ES, effect size; PLA, placebo; 10-RM, ten repetition maximum; WAT, water-only breakfast meal.

* Corresponding author: L. J. James, email L.James@lboro.ac.uk 
reported $^{(16)}$ that compared with a no-breakfast trial, an ecologically valid breakfast (containing $1.5 \mathrm{~g}$ carbohydrate/kg body mass) increased performance in four sets of back squat and four sets of bench press $2 \mathrm{~h}$ later. Collectively, these studies suggest that greater endogenous glycogen stores at the start of resistance exercise might increase performance.

However, there is another possible explanation, in that the results of these studies might be explained by the overtness of the methods used to manipulate pre-exercise nutritional state (i.e. conscious exercise/diet manipulation). Consequently, these previous studies might have been influenced by subjects' knowledge of, and preconceptions about, the intervention taking place. Indeed, Mears et al. ${ }^{(17)}$ recently demonstrated that a virtually energy-free placebo breakfast produces a similar increase in high-intensity cycling performance lasting approximately $20 \mathrm{~min}$ as a taste-/texture-matched high-carbohydrate ( $2 \mathrm{~g} / \mathrm{kg}$ body mass) breakfast, when compared with a wateronly control breakfast. This suggests that a pre-exercise meal/ breakfast might act as a placebo to enhance high-intensity aerobic performance, but whether these effects extend to resistance exercise (another high-intensity activity) and the related appetite effects are unknown.

Following an overnight fast, hunger is elevated, with consumption of breakfast suppressing hunger ${ }^{(18-20)}$, an effect that may be regulated by hormones involved in appetite regulation. Whether such appetite effects play a role in any performance responses to pre-exercise feeding is unknown, since most studies have not measured the two in combination, but Naharudin et $a l^{(16)}$ reported that the increased resistance exercise performance following breakfast occurred concurrently with a suppression in hunger. Therefore, if breakfast alters performance via a placebo, it is possible that the breakfast's effect on hunger might mediate the response.

Therefore, the aim of this study was to examine the effect of a pre-exercise high-carbohydrate breakfast meal on resistance exercise performance, compared with a texture- and tastematched placebo breakfast and a water control. This was to enable the physiological/metabolic effects of pre-exercise carbohydrate/energy intake to be separated from the potential psychological and/or appetite effects of eating a meal. It was hypothesised that the carbohydrate and placebo breakfast meals would increase resistance exercise performance compared with the control breakfast, with the carbohydrate breakfast meal also increasing resistance exercise performance compared the placebo breakfast meal.

\section{Experimental methods}

Subjects

This investigation was conducted according to the guidelines laid down in the Declaration of Helsinki, and all procedures were approved by the Loughborough University Ethics Approvals (Human Participants) Sub-Committee (R17-P073). Written informed consent was obtained from all subjects before participation. Twenty-two men experienced with resistance exercise (age 23 (sD 3) years, body mass 77.9 (sD 8.1) kg, height 1.75
(sD 0.05$) \mathrm{m}$, BMI $25 \cdot 2$ (sD $2 \cdot 0) \mathrm{kg} / \mathrm{m}^{2}$ ) completed this study. Twenty-four subjects commenced the study, but one withdrew due to an injury unrelated to the study, whilst another withdrew due to illness, meaning he was unable to complete his final trial. For enrolment into the study, subjects had to be nonsmokers, habitually consuming breakfast at least three mornings a week and to regularly include back squat and bench press as part of their weekly training. Subjects had 4.7 (SD 1.5) years resistance exercise experience and were performing 5 (SD 1) resistance training sessions/week, with 2 (SD 1) sessions/week of both back squat and bench press. All subjects reported eating breakfast $7 \mathrm{~d}$ /week. The sample size for this study was estimated from G*Power 3.0.10 software. Using an $\alpha$ of 0.05 , statistical power of 0.95 and data from a previous study ${ }^{(16)}$, it was estimated that twenty-two subjects would be sufficient to detect a $15 \%$ difference in back squat performance between trials. Subjects gave voluntary informed consent prior to participation.

\section{Study design}

The study examined the effect of a high-carbohydrate preexercise breakfast on resistance exercise performance, whilst controlling for any placebo effect associated with breakfast/ energy intake. Subjects visited the laboratory on five separate occasions: ten repetition maximum (10-RM) measurement; familiarisation trial and three experimental trials, during which subjects consumed a breakfast and approximately $2 \mathrm{~h}$ later performed an exhaustive bout of resistance exercise. The breakfasts used were a water-only control breakfast (WAT) and two low-energy viscous breakfasts, with the addition of no carbohydrate (PLA) or $1.5 \mathrm{~g}$ carbohydrate/kg body mass (CHO) as maltodextrin. Trials were arranged in a randomised order (randomisation by drawing trial orders for subjects out of a bag containing the six possible combinations), separated by $\geq 4 \mathrm{~d}$, and CHO and PLA breakfast meals were administered in a double-blind manner. Subjects were told the purpose of the experiment was to test two energy-containing breakfasts of different macronutrient compositions against a no-breakfast control trial. They were not aware that one of the breakfast trials contained virtually no energy.

\section{Preliminary visit and familiarisation trial}

After warming up (5-min cycling at $1.5 \mathrm{~W} / \mathrm{kg}$ body mass), subjects completed 10-RM testing of back squat and bench press, each of which was preceded by 5 min of self-selected exercisespecific warm-up. After some warm-up sets (self-selected), subjects were asked to perform their first attempt of each exercise at a weight close to their estimated 10-RM. The load increased incrementally thereafter until they could no longer complete ten repetitions. Subjects were given at least 3 min rest between sets. The last completed set of ten repetitions was termed subjects' 10-RM and was used to determine the study exercise workload. During a separate visit, subjects were fully familiarised with all procedures used in the experimental trials. 


\section{Pre-trial standardisation}

Subjects were given a diet/activity diary and were asked to record their habitual diet and activities for $2 \mathrm{~d}$ before their first experimental trial, replicating these patterns before subsequent trials. Subjects were asked to refrain from taking part in any vigorous activity or consumption of alcohol in the $2 \mathrm{~d}$ before trials.

\section{Experimental trials}

Subjects arrived at the laboratory after an overnight fast (10-13 h) at a time typical for them to consume breakfast (i.e. approximately 08.00-09.00 hours). Baseline measurements of body mass and subjective appetite were made, and after 15 min seated rest, a venous blood sample was collected. Subjects then consumed their allotted breakfast meal within $10 \mathrm{~min}$, with additional measures of subjective appetite taken immediately (i.e. $10 \mathrm{~min}$ ), $45 \mathrm{~min}$ and $105 \mathrm{~min}$ post-meal provision. Further venous blood samples were collected at $45 \mathrm{~min}$ and $105 \mathrm{~min}$. Subjects then completed the resistance exercise session described below.

\section{Breakfast meals}

During the two breakfast trials, subjects ate a semi-solid breakfast from a standard bowl using a standard spoon. The volume of the meal was $5 \mathrm{ml} / \mathrm{kg}$ body mass, of which $15 \%$ (i.e. $0.75 \mathrm{ml} / \mathrm{kg}$ body mass) was low-energy orange-flavoured squash (Double Strength Orange squash, Tesco), with the remainder made up of tap water. To this mixture, either $0 \mathrm{~g}$ carbohydrate $/ \mathrm{kg}$ body mass (PLA) or $1.5 \mathrm{~g}$ carbohydrate/ $\mathrm{kg}$ body mass of maltodextrin (Myprotein) was added and mixed thoroughly, before $0 \cdot 1 \mathrm{~g} / \mathrm{kg}$ body mass of xanthan gum (Myprotein) was added and the mixture blended to thicken the solution and enhance the perception of energy intake ${ }^{(21)}$. PLA and CHO breakfasts were taste-, texture- and colour-matched and were made the day before trials by an experimenter not involved in data collection. Additionally, $3 \mathrm{ml} / \mathrm{kg}$ body mass of tap water was consumed as a drink with meals. For the WAT trial, subjects consumed $8 \mathrm{ml} / \mathrm{kg}$ body mass of tap water to match the water content in PLA and CHO. The nutritional content of the breakfast meals is presented in Table 1.

At the end of the last experimental trial, subjects were informed of the contents of the breakfasts and the true aim of the study, before being asked if they could identify the

Table 1. Nutritional content of breakfast meals (Mean values and standard deviations)

\begin{tabular}{|c|c|c|c|c|c|c|}
\hline & \multicolumn{2}{|c|}{ WAT } & \multicolumn{2}{|c|}{ PLA } & \multicolumn{2}{|c|}{$\mathrm{CHO}$} \\
\hline & Mean & SD & Mean & SD & Mean & SD \\
\hline Protein (g) & 0 & 0 & 0.8 & 0.1 & 0.8 & 0.1 \\
\hline Carbohydrate (g) & 0 & 0 & $2 \cdot 4$ & 0.1 & $119 \cdot 2$ & $12 \cdot 4$ \\
\hline Fat $(\mathrm{g})$ & 0 & 0 & 0.6 & 0.1 & 0.6 & 0.1 \\
\hline Fibre $(\mathrm{g})$ & 0 & 0 & 5.4 & 0.6 & 5.4 & 0.6 \\
\hline Energy (kJ) & 0 & 0 & 122 & 13 & 2075 & 216 \\
\hline Water (ml) & 623 & 65 & 623 & 65 & 623 & 65 \\
\hline
\end{tabular}

WAT, water-only breakfast meal; PLA, placebo; $\mathrm{CHO}$, carbohydrate. breakfasts. If they answered yes, they were asked to say which was which.

\section{Resistance exercise performance}

Subjects performed the same warm-up described for the 10-RM testing, with the addition of two warm-up sets of ten repetitions at $30 \%$ and $60 \%$ of $10-\mathrm{RM}$ for each exercise. Subjects then performed four sets to failure at $90 \%$ of 10-RM. For each exercise, subjects performed a standardised lifting technique, with two spotters assisting them to reach the starting position for each set. For the squat, the bar was held across the back of the subject's shoulders and they started with their knees fully extended. They then lowered themselves until their thighs were parallel with the floor, before returning to the starting position. For bench press, subjects started with their elbows fully extended and lowered the bar until it lightly touched their chest, before returning to the starting position. Every repetition was counted in silence, and standardised verbal encouragement was given to the subjects throughout. All sets were separated by $3 \mathrm{~min}$ rest. Additional subjective appetite measures were made after the back squat and bench press exercise. Subjects consumed $0.5 \mathrm{ml} / \mathrm{kg}$ body mass of water immediately before the cycling warm-up, as well as before sets 1 and 3 of back squat and bench press.

\section{Subjective appetite sensations}

Throughout experimental trials, subjects rated their subjective sensations of hunger, fullness, light-headedness, tiredness, alertness and head soreness using paper-based visual analogue scales with written anchors of 'not at all'/'none at all' and 'extremely'/'a lot' placed 0 and $100 \mathrm{~mm}$, respectively ${ }^{(22)}$.

\section{Blood sampling and analysis}

For each blood sample, approximately $10 \mathrm{ml}$ blood was drawn by venepuncture from an antecubital/forearm vein after $15 \mathrm{~min}$ seated rest. Blood was dispensed into tubes (Sarstedt AG \& Co.) containing a clotting catalyst or EDTA $(1.6 \mathrm{mg} / \mathrm{ml})$ and centrifuged $\left(2400 \mathrm{~g}, 15 \mathrm{~min}, 4^{\circ} \mathrm{C}\right)$, and the resultant serum/plasma was stored at $-20^{\circ} \mathrm{C}$ until analysis. Plasma glucose was determined using a colorimetric assay and an autoanalyzer (Horiba Medical UK; CV 0.5\%), whilst serum insulin (Immunodiagnostic Systems; CV 5.1-12.1\%) and total concentrations of plasma total ghrelin (CV 1.7-1.8\%; Merck Millipore Ltd), total glucagon-like peptide-1 (CV 2.5-8.2\%; Merck Millipore Ltd) and total peptide tyrosine-tyrosine (CV 3.8-5.6\%; Merck Millipore Ltd) were determined using commercially available ELISA. Due to issues with blood collection for two subjects, blood samples were only collected from twenty subjects.

\section{Statistical analyses}

Data were analysed using SPSS (version 23.0; IBM Corp.). All data were checked for normality using a Shapiro-Wilk test. Data containing two factors were analysed using two-way repeated-measures ANOVA. Data containing one factor were analysed using one-way repeated-measures ANOVA. Where 
ANOVA indicated significant effects, post hoc Holm-Bonferroniadjusted paired sample $t$ tests or Holm-Bonferroni-adjusted Wilcoxon signed-rank tests were used, as appropriate. Statistical significance was set at $P<0 \cdot 05$, and data are presented as mean values and standard deviations. Cohen's $d z$ effect sizes (ES) were calculated for pairwise differences in performance, with $0.2,0.5$ and 0.8 considered the thresholds for small, medium and large ES, respectively.

\section{Results}

\section{Baseline measures and breakfast meal perception}

Subjects' baseline body mass was comparable between trials (WAT 77.4 (sD 8.3); PLA 77.5 (sD 8.4); CHO 77.4 (sD 8.4) kg; $P=0.671)$. Additionally, baseline hunger $(P=0.543)$, fullness $(P=0.961)$, alertness $(P=0.313)$, light-headedness $(P=0.904)$, head soreness $(P=0.894)$ and tiredness $(P=0.941)$ were similar between trials. Subjects rated the breakfast meals similarly (un)pleasant (WAT: 34 (sD 19) mm; PLA: 27 (sD 19) mm; CHO: 27 (sD 23) mm; $P=0.462)$ but rated PLA (73 (sD 19) $\mathrm{mm}$ ) and CHO (79 (sD 16) mm) more filling than WAT (34 (SD 24) $\mathrm{mm}$ $P<0.001)$, with no difference between PLA and CHO $(P=0 \cdot 176)$.

Thirteen subjects stated that they thought they could detect a difference between PLA and CHO breakfasts, with eleven correctly identifying the trials after they were notified what the trials were. Of these thirteen subjects, seven (back squat) and eight (bench press) performed better in the $\mathrm{CHO}$ trial compared with the PLA trial.

\section{Resistance exercise performance}

Total repetitions for back squat (Fig. 1(a)) were greater in PLA $(P<0.001 ; \mathrm{ES}=1.03$ large $)$ and $\mathrm{CHO}(P<0.001 ; \mathrm{ES}=1.25$ large $)$ than WAT, with no difference between PLA and $\mathrm{CHO}(P=1 \cdot 000$; $\mathrm{ES}=0.04)$. For back squat repetitions completed over the four individual sets (Fig. 1(b)), there were trial $(P<0 \cdot 001)$, time $(P<0.001)$ and interaction $(P=0.002)$ effects. Repetitions in sets $1(P<0.001)$ and $2(P<0.001)$ were greater in CHO and PLA compared with WAT, with no other differences between trials. For bench press, total repetitions were not different between trials (Fig. 2(a); $P=0 \cdot 130$ ). Pairwise comparisons between trials yielded ES of 0.31 (small) for WAT $v$. PLA, 0.63 (medium) for WAT $v$. CHO and 0.05 (trivial) for PLA $v$. CHO. There were trial $(P=0.01)$ and time $(P<0.001)$ effects, but no interaction effect $(P=0.234)$ for bench press repetitions completed over the four individual sets (Fig. 2(b)). Repetitions in set 1 were greater in CHO compared with WAT $(P=0 \cdot 039)$, with no other differences.

\section{Subjective appetite sensation}

There were trial $(P<0 \cdot 001)$, time $(P<0.001)$ and interaction $(P<0.001)$ effects for sensations of hunger and fullness (Fig. 3 ). Hunger was lower during PLA and CHO compared with WAT at all time points after breakfast $(P \leq 0 \cdot 002)$. Conversely, fullness was greater during PLA and CHO compared with WAT at all time
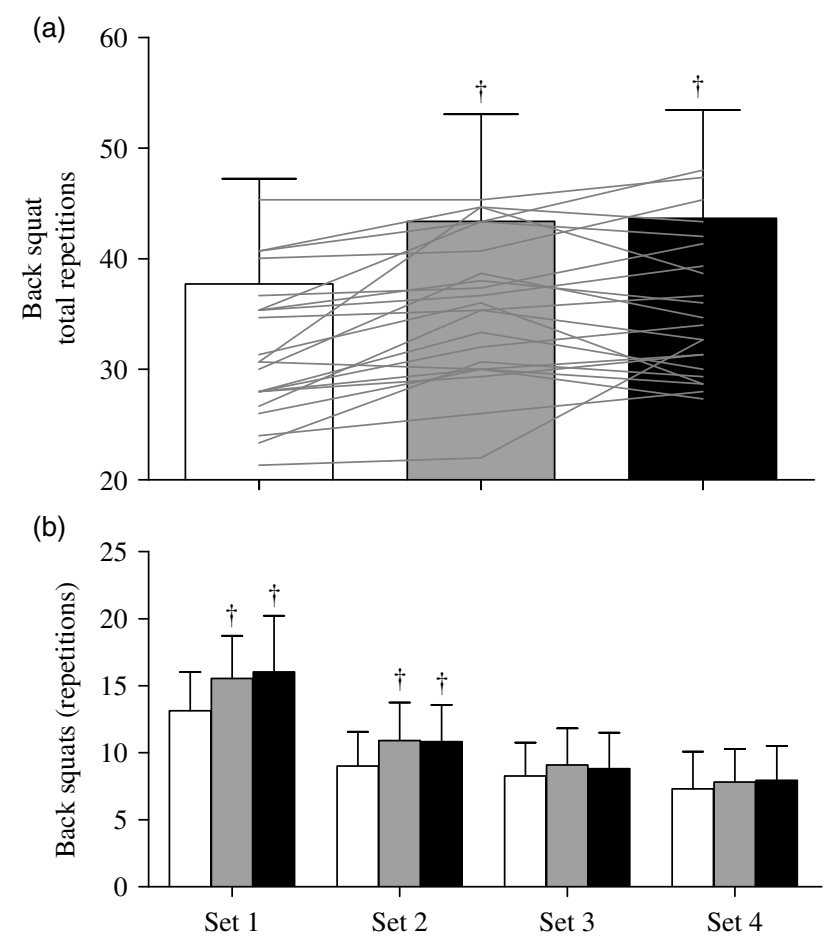

Fig. 1. Back squat repetitions in total over the four sets (a) and in each of the four sets (b) during the carbohydrate $(\mathrm{CHO})$, placebo (PLA) and water (WAT) trials. † Significantly different to WAT $(P<0.05)$. Values are means and standard deviations. $\square$, WAT; $\square$, PLA; $\square, \mathrm{CHO}$.

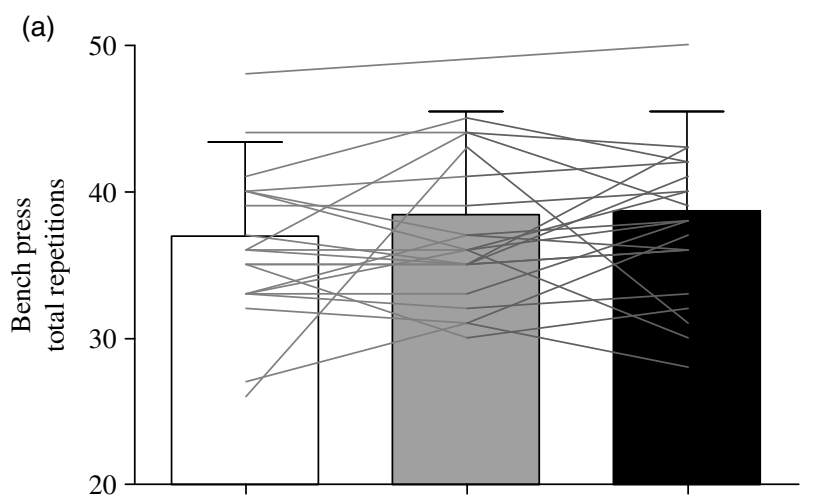

(b)

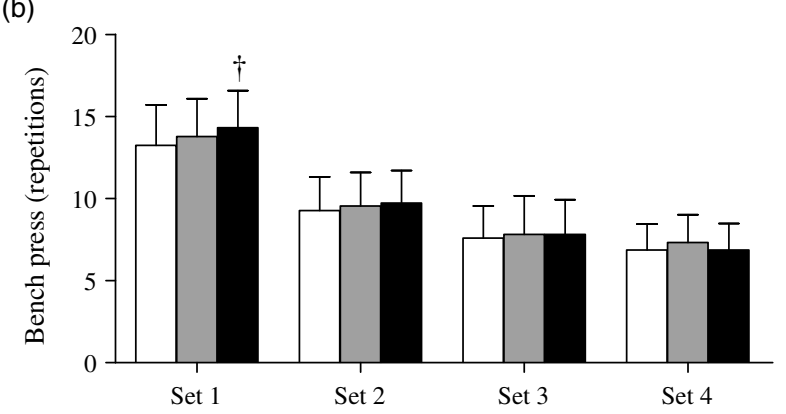

Fig. 2. Bench press repetitions in total over the four sets (a) and in each of the four sets (b) during the carbohydrate $(\mathrm{CHO})$, placebo (PLA) and water (WAT) trials. † Significantly different to WAT $(P<0.05)$. Values are means and standard deviations. $\square$, WAT; $\square$, PLA; $\square, \mathrm{CHO}$. 
(a)

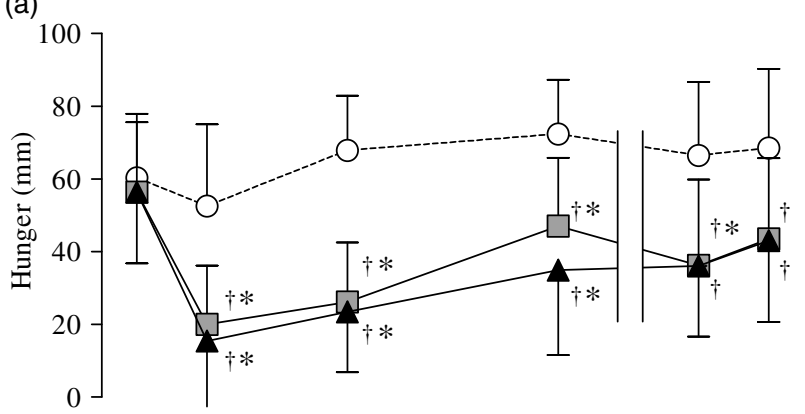

(b)

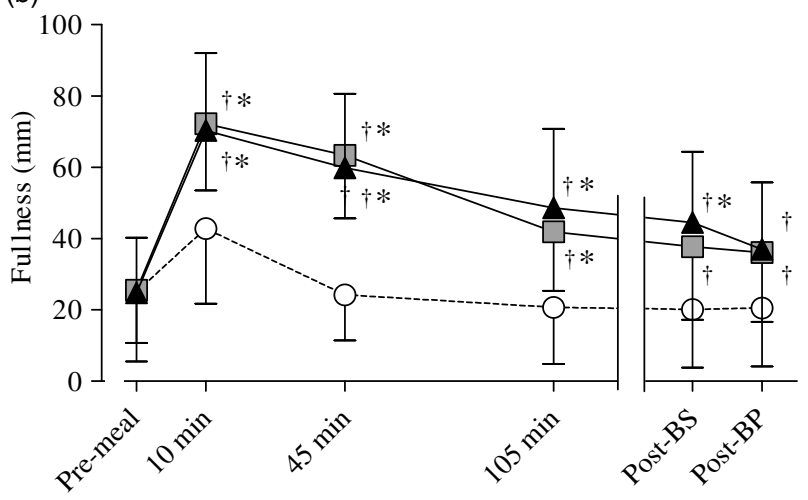

Fig. 3. Subjective ratings of hunger (a) and fullness (b) during the carbohydrate $(\mathrm{CHO})$, placebo (PLA) and water (WAT) trials. † Significantly different to WAT $(P<0.05) .{ }^{*}$ Significantly different from pre-meal $(P<0.05)$. Values are means and standard deviations. BS, back squat; BP, bench press. - $\mathrm{O}-$, WAT; $-\square-$, PLA $\triangle-$ CHO.

points after breakfast $(P \leq 0.027)$, with the exception of postbench press in $\mathrm{CHO}$, which tended to be greater $(P=0.055)$. Compared with pre-meal, hunger was reduced and fullness was increased at $10 \mathrm{~min}, 45 \mathrm{~min}$ and $105 \mathrm{~min}$ in CHO and PLA, and post-back squat in $\mathrm{CHO}(P<0 \cdot 05)$. Hunger and fullness were not different between PLA and CHO $(P \geq 0 \cdot 144)$. Whilst there was a main effect of time for sensations of alertness, light-headedness, head soreness and tiredness $(P<0 \cdot 001)$, there were no trial $(P \geq 0.319)$ or interaction $(P \geq 0.074)$ effects (data not shown).

\section{Blood analyses}

There were time $(P=0.003)$ and interaction $(P<0.001)$ effects, but no trial $(P=0.087)$ effect for plasma glucose concentration (Fig. 4(a)). Compared with pre-meal, plasma glucose was increased at $45 \mathrm{~min}$ during $\mathrm{CHO}$, returning to baseline at 105 min. Plasma glucose concentration did not change during WAT or PLA, and after Holm-Bonferroni correction, there were no between-trial differences. For serum insulin concentration, there were time $(P<0.001)$, trial $(P<0.001)$ and interaction $(P<0.001)$ effects. Insulin concentration (Fig. 4(b)) was increased at $45 \mathrm{~min}$ and $105 \mathrm{~min}$ compared with pre-meal in CHO only $(P<0.001)$ and additionally was greater at $45 \mathrm{~min}$ and $105 \mathrm{~min}$ in CHO compared with WAT and PLA $(P<0.001)$. For plasma ghrelin concentration (Fig. 5(a)), there were trial $(P<0.001)$ and interaction $(P=0.045)$ effects, but no time effect $(P=0 \cdot 206)$. Compared with pre-meal, plasma
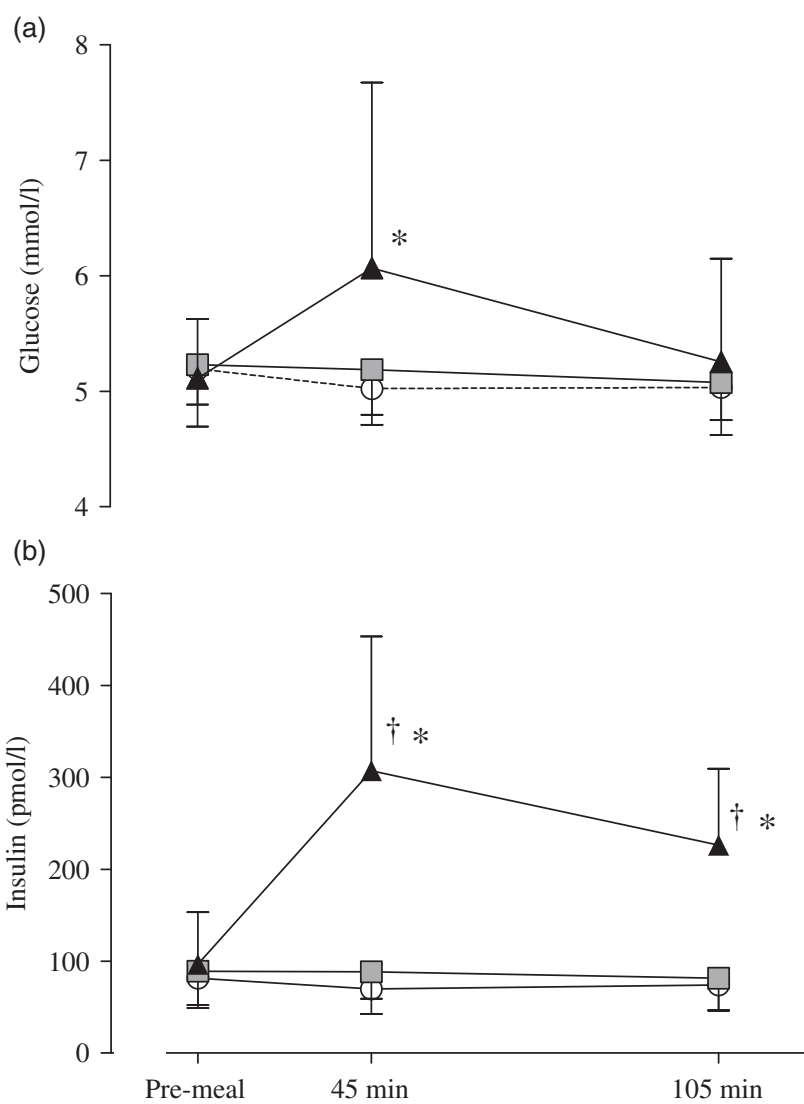

Fig. 4. Plasma glucose (a) and insulin (b) concentrations during the carbohydrate $(\mathrm{CHO})$, placebo (PLA) and water (WAT) trials. † Significantly different to WAT $(P<0.05)$. * Significantly different from pre-meal $(P<0.05)$. Values are means and standard deviations. - O-, WAT; $\square-\mathrm{PLA} ; \_$, $\mathrm{CHO}$.

ghrelin was decreased at $105 \mathrm{~min}$ in CHO only $(P=0 \cdot 010)$ and was lower in CHO compared with both WAT and PLA at $45 \mathrm{~min}(P<0.003)$ and $105 \mathrm{~min}(P<0.003)$. For plasma glucagon-like peptide-1 concentration (Fig. 5(b)), there were no trial $(P=0.940)$ or interaction $(P<0.391)$ effects, but there was a main effect of time $(P<0.001)$, with concentrations increasing at $45 \mathrm{~min}$ relative to pre-meal $(P=0.008)$. For plasma peptide tyrosine-tyrosine concentration (Fig. 5(c)), there were trial $(P=0.035)$ and time $(P=0.005)$ effects, but no interaction effect $(P=0.329)$. After Holm-Bonferroni correction, there were no differences between trials or from pre-meal.

\section{Discussion}

The aim of this study was to investigate the physiological and psychological effects of a pre-exercise high-carbohydrate breakfast meal on performance in a subsequent bout of resistance exercise in trained males. The main findings were that subjects completed more repetitions of back squat after consumption of virtually energy-free placebo and high-carbohydrate breakfast meals compared with the water-only control trial (PLA + 14.9\%; $\mathrm{CHO}+15.7 \% ; \quad P<0.001)$, with no difference between the placebo and carbohydrate breakfast meals $(P=1 \cdot 000)$. Smaller $(\mathrm{PLA}+3.9 \%$; $\mathrm{CHO}+4.7 \%)$, non-significant $(P=0 \cdot 130)$ effects 

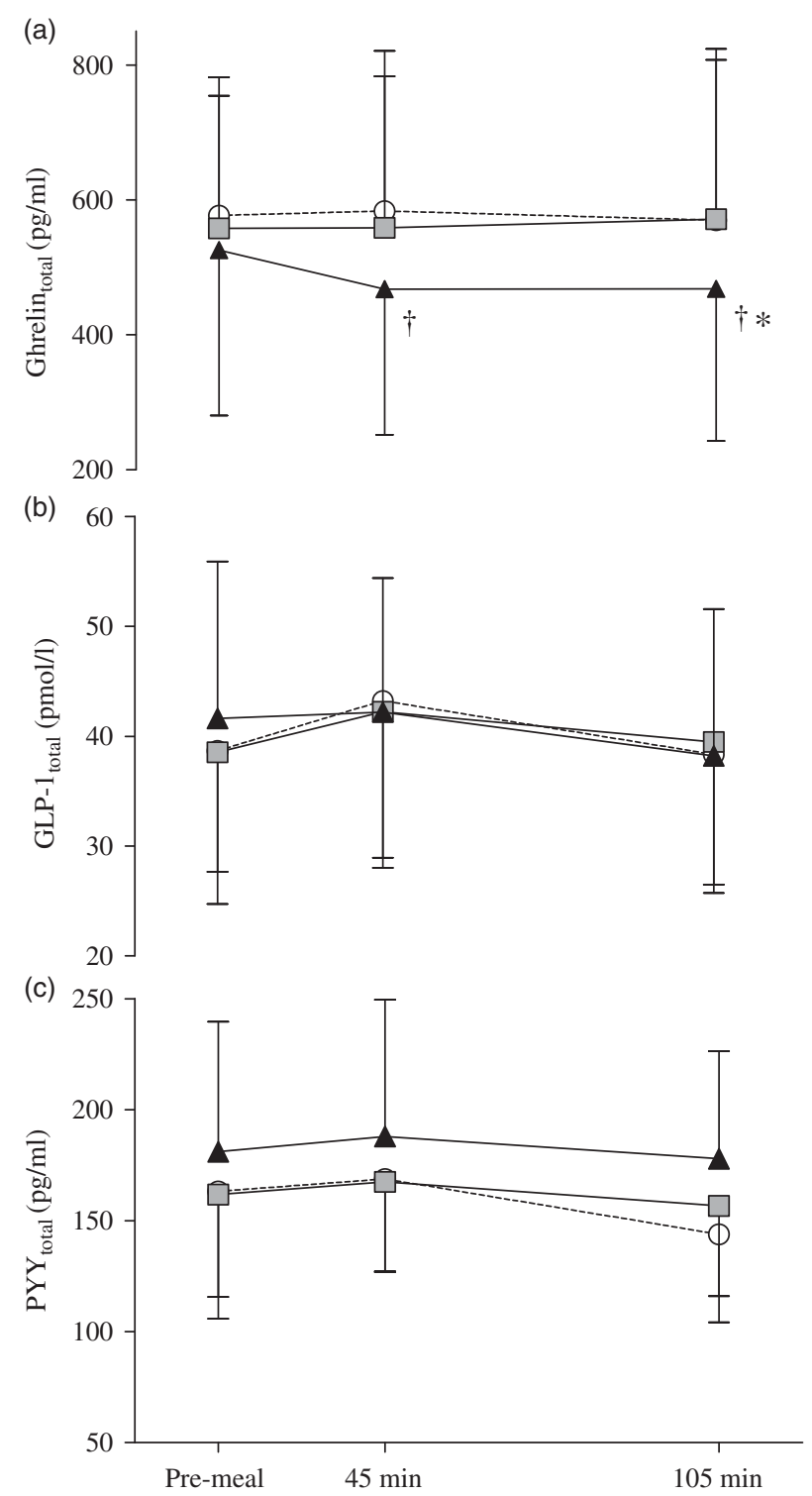

Fig. 5. Plasma ghrelin (a), glucagon-like peptide-1 (GLP-1) (b) and peptide tyrosine-tyrosine (PYY) (c) concentrations during the carbohydrate $(\mathrm{CHO})$, placebo (PLA) and water (WAT) trials. † Significantly different to WAT $(P<0.05)$. ${ }^{*}$ Significantly different from pre-meal $(P<0.05)$. Values are means and standard deviations. -O-, WAT; $\_-$PLA; $\_$- $\mathrm{CHO}$.

were observed for bench press. These results suggest that a pre-exercise breakfast meal likely influences resistance exercise performance via a psychological, rather than physiological effect, possibly acting as a placebo to enhance subsequent performance, at least in habitual breakfast consumers.

This study is the first to demonstrate that performance in a single bout of resistance exercise is enhanced when subjects believe they have consumed an energy-containing pre-exercise meal. This supports previous observations in a high-intensity cycling time trial lasting approximately $20 \mathrm{~min}^{(17)}$ and yields novel findings to optimise pre-exercise nutritional intake for resistance exercise. Importantly, the present study demonstrates that in well-rested men, consumption of a high-carbohydrate pre-exercise breakfast meal has no additional benefit over that of a placebo when four sets of both back squat and bench press are performed approximately $2 \mathrm{~h}$ later.

Previous research has mainly focused on the effects of a pre-exercise meal on endurance performance ${ }^{(2,23-25)}$. To our knowledge, only two studies have isolated the effect of a pre-exercise meal on resistance exercise performance ${ }^{(16,26)}$. Naharudin et al. ${ }^{(16)}$ observed that performance in four sets of back squat and four sets of bench press was both greater $2 \mathrm{~h}$ after a typical high-carbohydrate breakfast ( $1.5 \mathrm{~g}$ carbohydrate $/ \mathrm{kg}$ body mass) compared with a water-only breakfast. The increases in back squat (approximately 15\%) and bench press (approximately $6 \%$ ) performance in this previous study ${ }^{(16)}$ were almost identical to those observed in the present study (approximately $15 \%$ and $4 \%$, respectively), suggesting these previous findings are also likely to be explained by a placebo effect associated with pre-exercise feeding. Fairchild et al. ${ }^{(26)}$ reported similar performance responses in three repetitions of isokinetic knee extension/flexion for up to $90 \mathrm{~min}$ after consuming either a $75 \mathrm{~g}$ carbohydrate drink or a placebo drink. The failure of carbohydrate to enhance performance when delivered in a placebocontrolled manner in the study of Fairchild et al. ${ }^{(26)}$ further supports the theory that a pre-exercise meal/carbohydrate consumption might enhance resistance exercise performance via a placebo effect.

Muscle glycogen is an important fuel source for resistance exercise, and muscle glycogen depletion, particularly of type II muscle fibres, has been shown to decrease maximal strength ${ }^{(12)}$. The degree of muscle glycogen depletion during resistance exercise is related to the work completed ${ }^{(7)}$, and thus, if sufficient work is undertaken, muscle glycogen levels could become depleted to a level where performance capabilities are compromised ${ }^{(9)}$. In the $\mathrm{CHO}$ trial, plasma glucose was increased at $45 \mathrm{~min}$, whilst serum insulin was increased at $45 \mathrm{~min}$ and $105 \mathrm{~min}$, with no changes in the WAT and PLA trials. These findings suggest that the glucose in the $\mathrm{CHO}$ meal was absorbed and available for use before/during exercise. Carbohydrate feeding after an overnight fast has been shown to increase liver ${ }^{(27)}$ and, to a lesser extent, muscle ${ }^{(28,29)}$ glycogen stores. Although neither was measured in the present study, representing a limitation of our work, these results suggest that augmentation of these stores (at least typical meal carbohydrate intake) might not be necessary to maximise resistance exercise performance. It is possible that the amount of carbohydrate and/or the timing of the exercise in relation to the meal might have influenced the observed responses. Taylor et al. ${ }^{(29)}$ sequentially measured muscle glycogen content for $7 \mathrm{~h}$ after ingestion of a high-carbohydrate breakfast meal (289 g carbohydrate or approximately $4 \cdot 2 \mathrm{~g} / \mathrm{kg}$ body mass), observing peak increases (approximately $15 \%$ ) $4 \mathrm{~h}$ post-meal, with an approximately $3 \%$ increase at $2 \mathrm{~h}$ post-meal. Similarly, Chryssanthopoulos et al. ${ }^{(28)}$ reported an approximately $11 \%$ increase in muscle glycogen content $3 \mathrm{~h}$ after consuming a high-carbohydrate breakfast meal ( $2.5 \mathrm{~g}$ carbohydrate $/ \mathrm{kg}$ body mass). Therefore, in the present study, greater carbohydrate intake or allowing longer between the meal and exercise might have produced a slightly greater increase in muscle glycogen and possibly influenced performance. However, this seems unlikely, given that resistance exercise itself does not produce substantial glycogen 
depletion. In the present study, subjects rested for $48 \mathrm{~h}$ before each trial, meaning that muscle glycogen stores were likely to be high pre-meal, possibly accounting for the ineffectiveness of the carbohydrate breakfast meal to enhance performance. In many athletic settings, resistance training might occur only a few hours after another training session where glycogen may have been depleted. In this situation, it seems logical that addition of carbohydrate to the meal consumed before resistance exercise is more likely to be ergogenic, as it will also assist with recovery and replacement of glycogen used in the previous exercise bout ${ }^{(14)}$.

For endurance exercise lasting approximately $120 \mathrm{~min}$ at approximately $70 \% \quad \dot{\mathrm{V}}_{2} \max$, consuming carbohydrate $(>1.1 \mathrm{~g} / \mathrm{kg}$ body mass) in the $1-4 \mathrm{~h}$ before exercise appears to enhance exercise performance/capacity by approximately $9-15 \%{ }^{(28,30,31)}$. At these submaximal exercise intensities, muscle and liver glycogen depletion contribute to fatigue ${ }^{(10,25)}$, meaning that small differences in pre-exercise glycogen levels might influence performance ${ }^{(6)}$. In contrast, whilst muscle glycogen is used during resistance exercise, 3-6 sets of 6-12 repetitions of a single exercise only reduce muscle glycogen by $17-40 \%{ }^{(7,32-34)}$. Therefore, four sets of an exercise, as used in the present study, are unlikely to deplete local muscle glycogen to a level that would influence performance. The number of sets of each exercise performed in the present study was chosen to reflect current guidelines for those engaged in resistance training programmes, nominally $3-5$ sets per exercise ${ }^{(35)}$. Therefore, the present study suggests that any small increase in pre-exercise muscle glycogen caused by pre-exercise carbohydrate intake is unlikely to influence performance. Whether pre-exercise carbohydrate intake enhances performance in situations were substantially more than four sets are performed is not known and should be investigated in future studies. Furthermore, in practice, many resistance training sessions include more than two exercises and therefore in situations where multiple exercises using the same muscle groups are performed, pre-exercise carbohydrate intake may offer a benefit.

Liver glycogen has been shown to be an important fuel source in endurance exercise ${ }^{(36)}$, but its relevance to performance in resistance exercise is unknown. Liver glycogen is depleted during an overnight fast and although not measured in the present study, consumption of approximately $120 \mathrm{~g}$ carbohydrate in the $\mathrm{CHO}$ trial would likely have increased liver glycogen $^{(4)}$, whilst continued fasting in the PLA and WAT trials would likely produce a further decline in liver glycogen ${ }^{(27)}$. Therefore, exercise in the PLA and CHO trials likely commenced with very different liver glycogen levels. The finding that performance was not different between these trials therefore suggests that differences in liver glycogen within the range of normal daily fluctuations related to fasting and feeding are unlikely to influence performance in resistance exercise of this nature.

The results of the present study closely replicate the results of a previous study, where both high carbohydrate and placebo breakfasts, consumed $2 \mathrm{~h}$ before exercise, similarly enhanced high-intensity cycling performance lasting approximately $20 \min ^{(17)}$. To our knowledge, the present study and that of Mears et $a l{ }^{(17)}$ are the only studies to report the placebo effects of a pre-exercise meal, specifically breakfast. Breakfast is considered an important meal by many ${ }^{(37,38)}$, and as such it would be interesting to know whether the placebo effect of pre-exercise meals extends to other eating occasions. Regarding carbohydrate intake during exercise, a placebo effect has been observed during approximately $1 \mathrm{~h}$ cycling ${ }^{(39)}$, but not during approximately $3 \mathrm{~h}$ of cycling $^{(40)}$. Combined, these studies suggest that any placebo effect associated with energy (or carbohydrate) intake is possibly duration-dependent and is more likely to affect performance during exercise of shorter duration. It is possible that consuming a typical meal might produce a larger placebo effect than consumption of an atypical viscous meal of relatively low palatability. Although a limitation of the present study is that this effect cannot be discerned, the similarity in appetite and performance responses between this and our previous study ${ }^{(16)}$ suggests it is unlikely to be a major factor.

We used xanthan gum to increase the viscosity of the meals in the PLA and CHO trials to enhance the perception of energy intake. Previous research has shown that increasing the viscosity of a liquid/semi-solid meal increases subsequent satiety ${ }^{(21,41,42)}$. Consistent with these previous findings, we observed reduced hunger and increased fullness following the PLA and CHO meals compared with the WAT meal. Whilst there was a small amount of energy (approximately $122 \mathrm{~kJ}$ /approximately $29 \mathrm{kcal}$ ) in the PLA meal, contributed by xanthan gum and the orange squash, it seems unlikely this energy would cause these effects on subjective appetite, a notion that is supported by our results for the gut peptides peptide tyrosine-tyrosine, glucagon-like peptide- 1 and ghrelin. Peptide tyrosine-tyrosine and glucagonlike peptide-1, which are reported to exert anorexigenic effects $^{(43)}$, were not different between trials, despite greater hunger and lower fullness post-meal in WAT compared with PLA and CHO. Furthermore, ghrelin, reported to exert an orexigenic effect ${ }^{(44)}$, was reduced in the $\mathrm{CHO}$ trial only, and therefore we observed differences in ghrelin with (i.e. WAT $v$. CHO) and without (i.e. PLA $v$. CHO) differences in appetite, as well as no difference in ghrelin, despite a difference in appetite (i.e. WAT $v$. PLA). These results suggest that, at least after a single meal, there can be discordant responses for the subjective and physiological regulators of appetite and possibly questions the appetite regulating effects of these endocrine signals in such settings. Nonetheless, the differential response for ghrelin, as well as glucose and insulin, demonstrates that the CHO breakfast meal induced a physiological response. Interestingly, the differences in hunger/fullness between trials matched the differences in performance. Whether hunger can influence performance is not known and not possible to delineate in the present study, but it is possible that these subjective appetite sensations might mediate the effects of a pre-exercise breakfast/ meal on human performance.

Finally, the management of energy balance is of great importance for both athletes and recreational exercisers, particularly for those exercising to reduce or control body mass/fat. Omission of breakfast increases appetite in the morning ${ }^{(16,18-20)}$ and whilst, in some settings, this produces a small increase in energy intake at lunch ${ }^{(18,19)}$, total daily energy intake is generally reduced $^{(37,45,46)}$. However, breakfast omission also appears to reduce daily energy expenditure ${ }^{(47)}$, attenuating the energy 
deficit created by omission of breakfast. The results of the present study and that of Mears et al. ${ }^{(17)}$ suggest that a lowenergy high-viscosity pre-exercise breakfast might be an effective strategy to maintain exercise performance in situations of energy deficit, although whether this strategy has any effect on habitual daily energy expenditure remains to be seen. Furthermore, the present study observed reductions in appetite in the placebo breakfast that were similar to the carbohydratecontaining breakfast and comparable to responses reported previously following ecologically valid breakfasts ${ }^{(16,19)}$. Although speculative, this suggests that a low-energy high-viscosity placebo breakfast might also attenuate/prevent the increase in energy intake at a lunch meal. Future studies should seek to evaluate these effects.

In conclusion, the results of the present study demonstrate that performance in four sets of back squat exercise and four sets of bench press exercise was similarly enhanced by both placebo and carbohydrate-containing pre-exercise breakfast meals. This suggests that any performance effects of pre-resistance exercise energy/carbohydrate intake are likely caused by psychological effects, rather than any physiological/metabolic effect of the energy/carbohydrate content of the meal, at least in well-rested habitual breakfast consumers and that subjective appetite sensations, such as hunger, might be involved in these responses.

\section{Acknowledgements}

The authors thank all subjects who gave their time and commitment to complete this study. L. J. J. is part of the National Institute for Health Research (NIHR) Leicester Biomedical Research Centre, which is a partnership between University Hospitals of Leicester NHS Trust, Loughborough University and the University of Leicester. This report is independent research by the National Institute for Health Research. The views expressed in this publication are those of the authors and not necessarily those of the NHS, the National Institute for Health Research or the Department of Health.

This study is funded by the Research Management and Monitoring Research Fund - PG170-2014B and University of Malaya Research Grant - UM.0000215/HRU.OP/RU027-2015.

M. N. N. and L. J. J. formulated the research question and designed the study. M. N. N., J. A., T. T., H. R., C. O. and C. M. collected data. M. N. N., L. J. J., A. Y. analysed the data. M. N. N. and L. J. J. wrote the article with assistance from A. Y., D. J. C., C. J. H., S. A. M., J. A., T. T., H. R., C. O. and C. M.

L. J. J. has received funding for his research from Volac International Ltd, PepsiCo Inc., British Summer Fruits and The Collagen Research Institute, has performed consultancy work for Lucozade Ribena Suntory, has received conference fees from Danone Nutricia and PepsiCo Inc. and has received honoraria from PepsiCo Inc. In all cases, these payments have been paid to L. J J.'s employer and not to L. J. J.

\section{References}

1. Ralston GW, Kilgore L, Wyatt FB, et al. (2018) Weekly training frequency effects on strength gain: a meta-analysis. Sport Med Open 4, 36.
2. Ormsbee MJ, Bach CW \& Baur DA (2014) Pre-exercise nutrition: the role of macronutrients, modified starches and supplements on metabolism and endurance performance. Nutrients 6, 1782-1808.

3. Burke LM, Hawley JA, Wong SHS, et al. (2011) Carbohydrates for training and competition. J Sports Sci 29, Suppl. 1, S17-S27.

4. Nilsson LH \& Hultman E (1973) Liver glycogen in man - the effect of total starvation or a carbohydrate-poor diet followed by carbohydrate refeeding. Scand J Clin Lab Invest 32, 325-330.

5. Coyle EF, Coggan AR, Hemmert MK, et al. (1985) Substrate usage during prolonged exercise following a preexercise meal. J Appl Physiol 59, 429-433.

6. Chryssanthopoulos C, Williams C, Nowitz A, et al. (2002) The effect of a high carbohydrate meal on endurance running capacity. Int J Sport Nutr Exer Metab 12, 157-171.

7. Robergs RA, Pearson DR, Costill DL, et al. (1991) Muscle glycogenolysis during differing intensities of weight-resistance exercise. J Appl Physiol 70, 1700-1706.

8. Haff GG, Stone, MH, Warren BJ, et al. (1999) The effect of carbohydrate supplementation on multiple sessions and bouts of resistance exercise. J Strength Cond Res 13, 111-117.

9. Lambert CP \& Flynn, MG (2002) Fatigue during high-intensity intermittent exercise: application to bodybuilding. Sport Med 32, 511-522.

10. Ørtenblad N, Westerblad H \& Nielsen, J (2013) Muscle glycogen stores and fatigue. J Physiol 591, 4405-4413.

11. Coyle EF, Coggan AR, Hemmert MK, et al. (1986) Muscle glycogen utilization during prolonged strenuous exercise when fed carbohydrate. J Appl Physiol 61, 165-172.

12. Jacobs I, Kaiser P \& Tesch P (1981) Muscle strength and fatigue after selective glycogen depletion in human skeletal muscle fibers. Eur J Appl Physiol Occup Physiol 46, 47-53.

13. Leveritt M \& Abernethy PJ (1999) Effects of carbohydrate restriction on strength performance. J Strength Cond Res $\mathbf{1 3}$, $52-57$.

14. Oliver JM Almada AL, Van Eck L, et al. (2016) Ingestion of high molecular weight carbohydrate enhances subsequent repeated maximal power: a randomized controlled trial. PLOS ONE 11, $\mathrm{e} 0163009$.

15. Mitchell JB, DiLauro PC, Pizza F, et al. (1997) The effect of preexercise carbohydrate status on resistance exercise performance. Int J Sport Nutr 7, 185-196.

16. Naharudin MN, Yusof A, Shaw H, et al. (2019) Breakfast omission reduces subsequent resistance exercise performance. J Strength Cond Res 7, 1766-1772.

17. Mears SA, Dickinson K, Bergin-Taylor K, et al. (2017) Perception of breakfast ingestion enhances high intensity cycling performance. Int J Sports Physiol Perform 13, 504-509.

18. Chowdhury EA, Richardson JD, Tsintzas K, et al. (2016) Effect of extended morning fasting upon ad libitum lunch intake and associated metabolic and hormonal responses in obese adults. Int J Obes 40, 305-311.

19. Clayton DJ, Barutcu A, Machin C, et al. (2015) Effect of breakfast omission on energy intake and evening exercise performance. Med Sci Sports Exerc 47, 2645-2652.

20. Clayton DJ, Stensel DJ \& James LJ (2016) Effect of breakfast omission on subjective appetite, metabolism, acylated ghrelin and GLP-17-36 during rest and exercise. Nutrition 32, 179-185.

21. Fiszman S \& Varela P (2013) The role of gums in satiety/ satiation. A review. Food Hydrocoll 32, 147-154.

22. Flint A, Raben A, Blundell JE, et al. (2000) Reproducibility, power and validity of visual analogue scales in assessment of appetite sensations in single test meal studies. Int J Obes $\mathbf{2 4}$, $38-48$. 
23. Neufer PD, Costill DL, Flynn MG, et al. (1987) Improvements in exercise performance: effects of carbohydrate feedings and diet. J Appl Physiol 62, 983-988.

24. Coggan AR \& Coyle EF (1987) Reversal of fatigue during prolonged exercise by carbohydrate infusion or ingestion. J Appl Physiol 63, 2388-2395.

25. Coyle EF \& Coggan AR (1984) Effectiveness of carbohydrate feeding in delaying fatigue during prolonged exercise. Sports Med 1, 446-458.

26. Fairchild TJ, Dillon P, Curtis C, et al. (2016) Glucose ingestion does not improve maximal isokinetic force. J Strength Cond Res 30, 194-199.

27. Bawden SJ, Stephenson MC, Ciampi E, et al. (2014) A low calorie morning meal prevents the decline of hepatic glycogen stores: a pilot in vivo ${ }^{13} \mathrm{C}$ magnetic resonance study. Food Funct 5, 2237-2242.

28. Chryssanthopoulos C, Williams C, Nowitz A, et al. (2004) Skeletal muscle glycogen concentration and metabolic responses following a high glycaemic carbohydrate breakfast. J Sports Sci 22, 1065-1071.

29. Taylor R, Price TB, Katz LD, et al. (1993) Direct measurement of change in muscle glycogen concentration after a mixed meal in normal subjects. Am J Physiol 265, E224-E229.

30. Sherman WM, Brodowicz G, Wright DA, et al. (1989) Effects of $4 \mathrm{~h}$ preexercise carbohydrate feedings on cycling performance. Med Sci Sport Exerc 21, 598-604.

31. Sherman WM, Peden MC \& Wright DA (1991) Carbohydrate feedings $1 \mathrm{~h}$ before exercise improves cycling performance. Am J Clin Nutr 54, 866-870.

32. Tesch PA, Ploutz-Snyder LL, Yström L, et al. (1998) Skeletal muscle glycogen loss evoked by resistance exercise. J Strength Cond Res 12, 67-73.

33. MacDougall JD, Ray S, Sale DG, et al. (1999) Muscle substrate utilization and lactate production during weightlifting. Can J Appl Physiol 24, 209-215.

34. Haff GG, Koch AJ, Potteiger JA, et al. (2000) Carbohydrate supplementation attenuates muscle glycogen loss during acute bouts of resistance exercise. Int J Sport Nutr Exerc Metab 10, 326-339.
35. Ratamess NA (2009) American College of Sports Medicine position stand: progression models in resistance training for healthy adults. Med Sci Sports Exerc 41, 687-708.

36. Gonzalez JT, Fuchs CJ, Betts JA, et al. (2016) Liver glycogen metabolism during and after prolonged endurance-type exercise. Am J Physiol Endocrinol Metab 311, E543-E553.

37. Clayton DJ \& James LJ (2015) The effect of breakfast on appetite regulation, energy balance and exercise performance. Proc Nutr Soc 75, 319-327.

38. Betts JA, Chowdhury EA, Gonzalez JT, et al. (2016) Is breakfast the most important meal of the day? Proc Nutr Soc 75, 464-474.

39. Clark VR, Hopkins WG, Hawley JA, et al. (2000) Placebo effect of carbohydrate feedings during a 40-km cycling time trial. Med Sci Sports Exerc 32, 1642-1647.

40. Hulston CJ \& Jeukendrup AE (2009) No placebo effect from carbohydrate intake during prolonged exercise. Int J Sport Nutr Exerc Metab 19, 275-284.

41. Marciani L, Gowland PA, Spiller RC, et al. (2000) Gastric response to increased meal viscosity assessed by echo-planar magnetic resonance imaging in humans. J Nutr 130, 122-127.

42. Marciani L, Gowland PA, Spiller RC, et al. (2001) Effect of meal viscosity and nutrients on satiety, intragastric dilution, and emptying assessed by MRI. Am J Physiol Liver Physiol 280, G1227-G1233.

43. Batterham RL, Cowley MA, Small CJ, et al. (2002) Gut hormone PYY3-36 physiologically inhibits food intake. Nature $\mathbf{4 1 8}$, 650-654.

44. Cummings DE \& Overduin J (2007) Review series gastrointestinal regulation of food intake. J Clin Invest 117, 13-23.

45. Gonzalez JT, Veasey RC, Rumbold PLS, et al. (2013) Breakfast and exercise contingently affect postprandial metabolism and energy balance in physically active males. Br J Nutr 110, 721-732.

46. Edinburgh RM, Hengist A, Smith HA, et al. (2019) Skipping breakfast before exercise creates a more negative 24-hour energy balance: a randomized controlled trial in healthy physically active young men. J Nutr 149, 1326-1334.

47. Betts JA, Richardson JD, Chowdhury EA, et al. (2014) The causal role of breakfast in energy balance and health: a randomized controlled trial in lean adults. Am J Clin Nutr 100, 539-547. 